\title{
Das Thüringer Berufswahlkompetenzmodell
}

In diesem Kapitel wird die Auswahl des Berufswahlkompetenzmodells von Driesel-Lange et al. (2010) als theoretische Grundlage der empirischen Studien in Kapitel 10 bis 12 entfaltet. Im ersten Teilkapitel wird dafür das Modell mit seinen theoretischen Bezügen, der Aufteilung des Berufswahlprozesses in Phasen und seiner multidimensionalen Perspektive auf Berufswahlkompetenz erörtert. Das zweite Teilkapitel erläutert den diagnostischen Fragebogen zur Erfassung von Berufswahlkompetenz von Kaak, Driesel-Lange, Kracke und Hany (2013). Für einen Überblick über die aktuelle empirische Forschung hinsichtlich des Berufswahlkompetenzmodells wird insbesondere auf die Kapitel 11 und 12 verwiesen. Im dritten Teilkapitel wird das Berufswahlkompetenzmodell mit den in Abschnitt 4.1 vorgestellten Entwicklungsmodellen und den Kompetenz- und Ressource-Modellen aus Abschnitt 5.2 verglichen, um seine Auswahl für die vorliegende Arbeit zu begründen.

\subsection{Theoretische Bezüge, Phasenbezug und Multidimensionalität}

Das Berufswahlkompetenzmodell bildet das Kernstück des Thüringer Berufsorientierungsmodells, welches von Driesel-Lange et al. (2010) als ganzheitliches, multidimensionales Modell zur Unterstützung theoriegeleiteter, bedarfsorientierter beruflicher Orientierung an Schulen entwickelt wurde (Driesel-Lange et al., 2020). Zwei weitere Teilmodelle ergänzen das Berufswahlkompetenzmodell. Das Modell zur Kompetenzvermittlung formuliert die Rolle von Lehrkräften in der schulischen Begleitung beruflicher Orientierung und die Anforderungen, die in diesem Rahmen an sie gestellt werden. Das Implementationsmodell wiederum beschreibt Grundsätze einer organisatorischen Umsetzung (Driesel-Lange et al., 
2020). Hintergrund, Ziele und Aufbau dieser beiden Teilmodelle werden von Driesel-Lange et al. (2020) ausführlich erläutert. Seit seiner erstmaligen Veröffentlichung im Jahr 2010 wurde das Modell sukzessive mit Blick auf neuere Entwicklungen in der internationalen Theoriebildung (Blustein, 2017) sowie in Rückkopplung zu neueren empirischen Befunden (Brüggemann, Driesel-Lange \& Weyer, 2017) und Erkenntnissen aus der Praxis (Kunz \& Schreiber, 2017; Lipowski et al., 2015b) weiterentwickelt (Driesel-Lange et al., 2020; Driesel-Lange, Kracke, Hany \& Schindler, 2013).

Die folgende Beschreibung des Berufswahlkompetenzmodells bezieht sich auf seine neueste Fassung und lehnt sich an die Darstellung von Driesel-Lange et al. (2020) an. Das Modell bettet verschiedene wissenschaftliche Perspektiven auf berufliche Entwicklung ein, und zwar die des individuellen Entwicklungsprozesses, der Selbststeuerung, der Ergebnis- und Kompetenzorientierung, des Expertise-Erwerbs und die des sozialen Kontexts als Einflussfaktor (DrieselLange et al., 2020). Über diese Aspekte knüpft das Berufswahlkompetenzmodell, wie im Folgenden entfaltet wird, an internationale Theorien an. Sie können darüber hinaus als wichtige Grundsätze erfolgreicher beruflicher Entwicklung in der Adoleszenz verstanden werden (Herr et al., 2004; Hirschi, 2010b).

Der Berufswahlprozess wird im Thüringer Berufsorientierungsmodell als ein höchst individueller, durch die Jugendlichen selbstgesteuerter Prozess verstanden (Driesel-Lange et al., 2020). Dabei stellen die berufliche Entwicklung und die Bildung einer beruflichen Identität das Ergebnis eines sozialen Lernprozesses dar (Mitchell \& Krumboltz, 1990). In diesem Lernprozess erwerben Jugendliche durch Beobachtung und Erprobung, beispielsweise von Arbeitsmethoden und Tätigkeiten, berufswahlrelevante Erfahrungen und Kompetenzen. Diese Expertise bildet die Grundlage, um die eigenen Interessen im Vergleich zu den Anforderungen eines Berufs zu reflektieren und eine begründete Berufswahl zu treffen. Erfahrungen mit der Berufs- und Arbeitswelt, die aus dem Lernprozess resultieren, unterstützen zudem den Übergang von der Schule in nachschulische Kontexte (Herzog et al., 2006).

In diesem Prozess werden die Jugendlichen auch durch ihre Umwelt, u. a. in ihren beruflichen Präferenzen beeinflusst (Gottfredson, 2002, siehe Abschnitt 3.2). Gleichzeitig übt das soziale Umfeld eine unterstützende Funktion in der beruflichen Entwicklung aus, wie Schindler (2012) und Ginevra, Nota und Ferrari (2015) für die Rolle der Lehrkräfte, respektive der Eltern empirisch nachzeichnen konnten. Auch der Life Designing-Ansatz fußt auf der Annahme, dass der Wissenserwerb und seine Verarbeitung in einer Ko-Konstruktion durch den sozialen Austausch entstehen (Savickas et al., 2009). 


\section{Phasen beruflicher Entwicklung im Berufswahlkompetenzmodell}

Die für das Berufswahlkompetenzmodell maßgebliche Annahme einer individuellen, selbstgesteuerten Entwicklung und das Ziel, Jugendliche bei der individuellen Gestaltung ihrer „Geschichte“ (,story“) (Kunz \& Schreiber, 2017; Patton \& McMahon, 2017) zu unterstützen, knüpfen an Savickas (2005) konstruktivistische Theorie der Laufbahnentwicklung an (siehe Abschnitt 5.2). Die entwicklungspsychologische Perspektive auf Berufswahl als einen Prozess individueller Entwicklung durchwebt alle Bereiche des Berufswahlkompetenzmodells. Entsprechend durchlaufen Jugendliche in ihrem individuellen Tempo vier entwicklungstypische Phasen, nämlich die des Einstimmens, des Erkundens, des Entscheidens und des Erreichens (Driesel-Lange et al., 2020). Jeder Phase wohnen phasentypischen Entwicklungsaufgaben und Ziele inne. Wie Abbildung 6.1 darstellt, erwerben die Jugendlichen im Laufe der vier Phasen zwölf Aspekte von Berufswahlkompetenz - sogenannte Berufswahlkompetenzfacetten -, die im Verlauf des aktuellen Kapitels genauer beschrieben werden.

Empirische Studien wie die von Driesel-Lange und Kracke (2017) konnten unterschiedliche Entwicklungsstände der Berufswahlkompetenz innerhalb einer Jahrgangsstufe zeigen und damit die theoretische Annahme individueller Entwicklungstempi auch empirisch untermauern.

Das Entwicklungsziel der ersten Phase - das Einstimmen - besteht vornehmlich darin, die Berufswahl als eine für sich selbst bedeutsame Aufgabe zu begreifen und anzunehmen. Jugendliche sollen sich in dieser Phase mit ihren eigenen Interessen, Werten und Zielen beschäftigen, um diese in ihrer weiteren beruflichen Orientierung berücksichtigen zu können. Auch hier nimmt das Berufswahlkompetenzmodell wiederum Bezug auf den individuellen Charakter beruflicher Entwicklung. Neben dem Anerkennen der Berufswahl als einer wichtigen Aufgabe, lernen Jugendliche in dieser Phase, die auch den Anfangspunkt ihrer beruflichen Orientierung markiert, idealerweise die für eine langfristig erfolgreiche Berufswahl wesentlichen Fähigkeiten kennen (Driesel-Lange et al., 2020). Ein wesentliches Ziel der zweiten Entwicklungsphase wird bereits durch ihren Namen - das Erkunden - deutlich. In dieser Phase sollen Jugendliche systematisch und gezielt ihre eigenen Interessen erkunden und Informationen zu Berufen, Ausbildungswegen und Zugangsvoraussetzungen sammeln. Maßgabe dabei ist eine möglichst breit angelegte Suche, um auch bisher unbekannte Berufsfelder, in denen die eigenen Interessen und Stärken anwendbar sind, zu entdecken. Dieser Suche folgt nach einer ersten Reflektion eine eingegrenzte, tiefergehende Suche (Driesel-Lange et al., 2020). Das Erkunden bezieht sich außerdem auf die gezielte und systematische Suche nach Situationen, um praktische Erfahrungen in der 


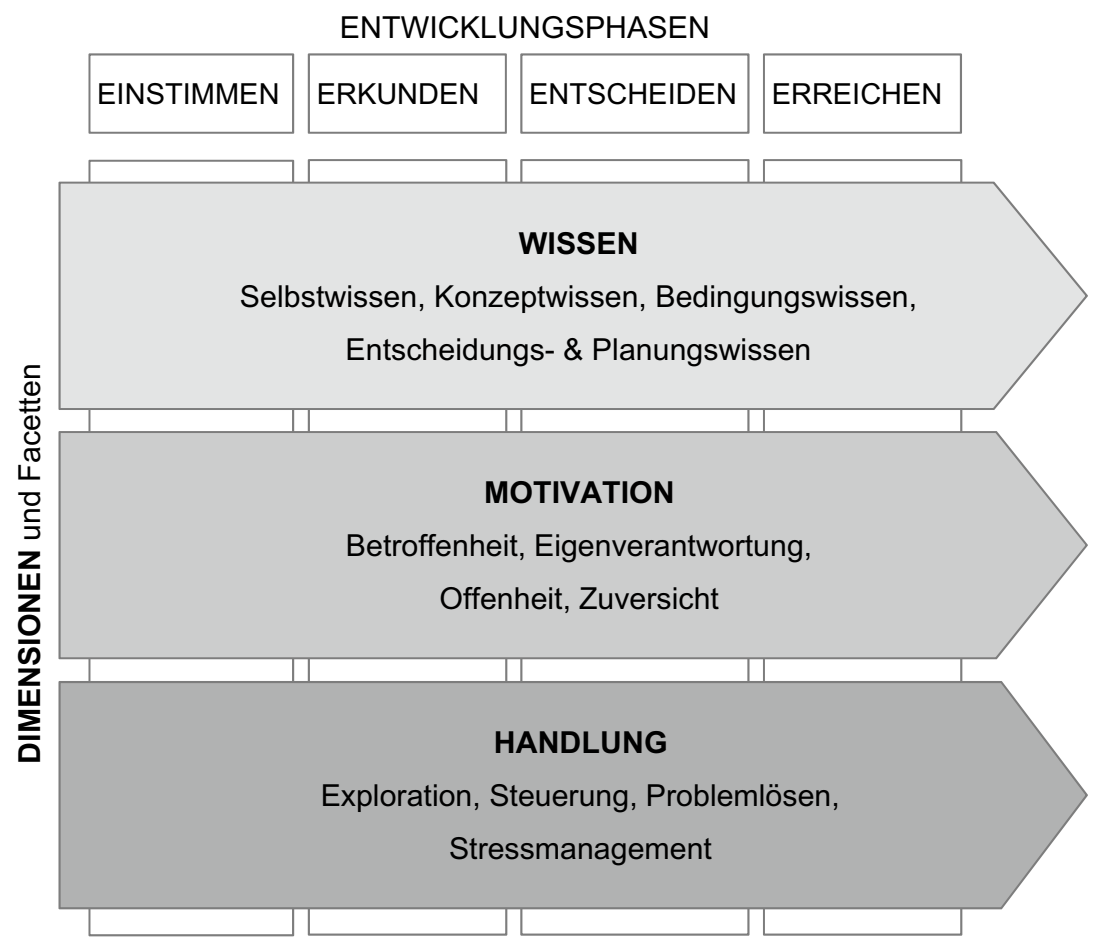

Abbildung 6.1 Das Berufswahlkompetenzmodell von Driesel-Lange et al. (2020)

Arbeitswelt zu sammeln. Der Expertise-Erwerb ist in dieser Phase also ein zweites zentrales Ziel. Auch hier zeigt sich die Anschlussfähigkeit des Modells an internationale Theorien wie die des Life Designings (Savickas, 2012). Denn die Existenz von Expertise, im Sinne von Erfahrung, stellt eine Grundlage dar, um diese als Referenzrahmen für anschließende berufliche Entscheidungen fruchtbar zu machen und die eigene berufliche Geschichte gestalten zu können. Aus der Reflektion der eigenen praktischen Erfahrungen entsteht in Kombination mit der Informationssuche zu Berufsbildern und Tätigkeiten ein realistischeres Bild der Berufs- und Arbeitswelt. Im weiteren Verlauf sollen diese Informationen und Erfahrungen als Grundlage einer fundierten Entscheidung dienen. Die dritte Entwicklungsphase - das Entscheiden - stellt eine Zeit des Abwägens verschiedener Optionen und des Konkretisierens des nachschulischen Anschlusses dar. Jugendliche sollen die in Betracht kommende, engere Auswahl an Berufen, ihre eigenen 
Interessen und Fähigkeiten, sowie ihre Werte und den gewünschten Lebensstil kritisch prüfen und miteinander vergleichen, um schließlich eine Entscheidung ableiten zu können. Die vierte Phase - das Erreichen - wird von dem Einleiten konkreter Umsetzungsschritte bestimmt. Dazu gehört insbesondere die Bewerbung um einen Ausbildungs- oder Studienplatz. Des Weiteren sollen sich die Jugendlichen in dieser Phase mit ihrer neuen beruflichen Rolle beschäftigen. Es gilt die anstehenden Veränderungen und potentiellen privaten, sozialen, finanziellen, organisatorischen Herausforderungen frühzeitig zu erkennen, um potentielle Lösungen zu antizipieren (Driesel-Lange et al., 2020).

\section{Erwerb von Berufswahlkompetenz}

Eine weitere Gelingensbedingung erfolgreicher beruflicher Entwicklung und gleichzeitig zentraler Aspekt des Berufswahlkompetenzmodells ist der Erwerb berufswahlrelevanter Kompetenzen. Dabei wird Weinerts (2001b) Verständnis von Kompetenz als Resultat von Lern- und Entwicklungsprozessen zugrunde gelegt (Driesel-Lange et al., 2020, siehe Abschnitt 5.1).

Driesel-Lange et al. (2020) verwenden Berufswahlkompetenz als einen Oberbegriff für insgesamt zwölf berufswahlrelevante Aspekte. Diese zwölf Facetten von Berufswahlkompetenz können als kognitive Fähigkeiten, motivationale Einstellungen und Handlungsfunktionen charakterisiert werden. Entsprechend der situativen Herausforderungen, die sich aus den Aufgaben der oben beschriebenen vier Phasen ergeben, verändert sich die Relevanz der einzelnen Berufswahlkompetenzfacetten (Driesel-Lange et al., 2020). Abbildung 6.2 verbildlicht die Relevanz der einzelnen Berufswahlkompetenzfacetten in den vier Entwicklungsphasen.

Demgemäß unterscheidet das Modell drei Dimensionen, nämlich Wissen, Motivation und Handlung. Nach Hartig und Klieme (2006) lässt sich das Berufswahlkompetenzmodell aufgrund der Einteilung der Facetten in übergeordnete Dimensionen als Kompetenzstrukturmodell bezeichnen. Im Folgenden werden die zwölf Facetten entlang der drei genannten Dimensionen aufgeschlüsselt und erläutert.

\section{Die Wissensdimension}

Die erste Wissensfacette, das Selbstwissen, umfasst die Kenntnisse der Jugendlichen über ihre eigenen Fähigkeiten, Talente und Potentiale, aber auch ihre Interessen, Lebensziele, Werte und Einstellungen. Das Konzeptwissen, die zweite Facette in der Wissensdimension, bezieht sich auf das Verständnis der Arbeitsund Berufswelt. Dazu gehören allgemeine Kenntnisse zu Branchen und Berufsfeldern sowie spezifische Kenntnisse typischer Arbeitsabläufe eines Berufs. Des 


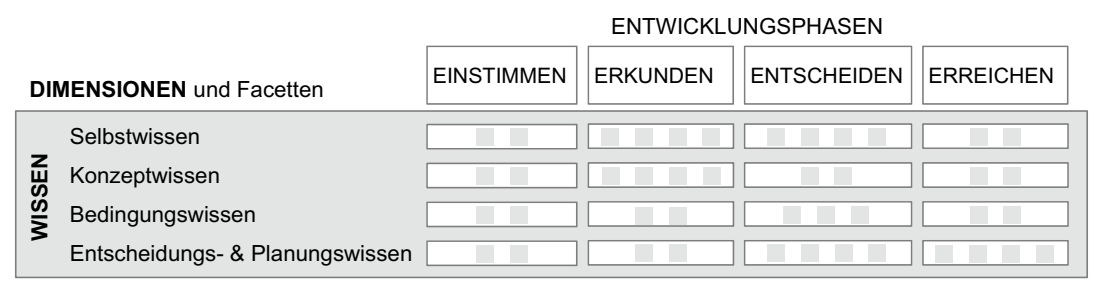

\begin{tabular}{|c|c|c|c|c|c|}
\hline \multirow{4}{*}{ 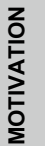 } & Betroffenheit & 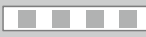 & 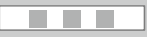 & प्वप्र & $\square \square$ \\
\hline & Eigenverantwortung & 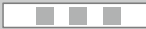 & 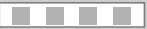 & 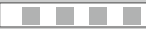 & 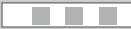 \\
\hline & Offenheit & $\square \square$ & 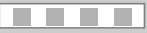 & $\square \square \square$ & $\begin{array}{ll}\square \square \square \\
口\end{array}$ \\
\hline & Zuversicht & $+\mathrm{n}$ & $\square$ & $\mathrm{F}^{\mathrm{n}}$ & 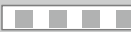 \\
\hline
\end{tabular}

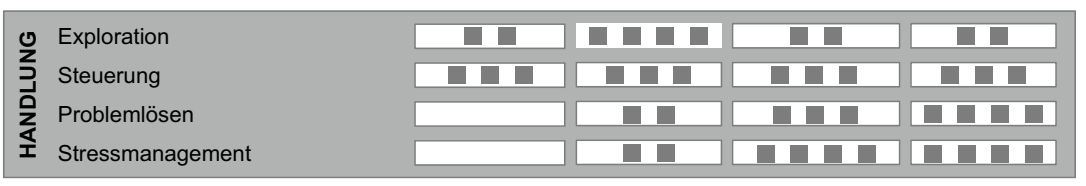

Abbildung 6.2 Relevanz der einzelnen Berufswahlkompetenzfacetten in der beruflichen Entwicklung. (Darstellung in Anlehnung an Driesel-Lange et al., 2020)

Weiteren bedeutet Konzeptwissen, Bildungseinrichtungen wie Berufs- und Hochschulen mit ihren verschiedenen Bildungsgängen zu kennen. Die dritte Facette dieser Dimension, das Bedingungswissen, bezeichnet das Wissen über akademische, strukturelle, organisatorische und finanzielle Zugangsvoraussetzungen für eine Ausbildung bzw. für die Ausübung eines bestimmten Berufs. Das Wissen um direkte und alternative Einmündungsmöglichkeiten, beispielsweise durch den Erwerb der Hochschulreife auf dem zweiten Bildungsweg, sowie das Bewusstsein für Konfliktpotentiale in der Vereinbarkeit des gewählten Berufs und des angestrebten Lebensmodells, zählen auch zum Bedingungswissen (DrieselLange et al., 2020). Als Planungs- und Entscheidungswissen ${ }^{1}$ wird die Fähigkeit bezeichnet, die eigene berufliche Entwicklung in Bezug auf zu steckende Ziele, bestehende Entwicklungsmöglichkeiten und potentielle Hindernisse hin zu antizipieren. Planung- und Entscheidungswissen befähigt weiter dazu, Etappenziele zu formulieren und diese in einen realistischen Zeitplan zu fassen. Das übergeordnete Ziel kann zudem kritisch in Bezug auf eine Realisierbarkeit hin überprüft werden.

\footnotetext{
${ }^{1}$ Die ursprüngliche Planungs- und Entscheidungskompetenz wurde mit der Überarbeitung des Berufswahlkompetenzmodells in Planungs- und Entscheidungswissen umbenannt (DrieselLange et al., 2020).
} 
Mittels ihres Planungs- und Entscheidungswissens können Jugendliche auch alternative Wege zu ihrem Ziel bzw. Alternativziele entwickeln (Driesel-Lange et al., 2020).

\section{Die motivationale Dimension}

Auch die motivationale Dimension besteht aus vier Facetten. Wie Driesel-Lange et al. (2020) feststellen, handelt es sich bei der Beschäftigung mit der eigenen beruflichen Zukunft und den daraus entstehenden, teilweise weit entfernten Konsequenzen, um eine bedeutende und zugleich komplexe Aufgabe, die entsprechende motivationale Einstellungen erfordert. Dabei spielt die erste motivationale Facette, die Betroffenheit als die Bereitschaft, sich mit der eigenen beruflichen Zukunft kritisch und aktiv beschäftigen zu wollen, eine wichtige Rolle. Darüber hinaus müssen Jugendliche die Überzeugung entwickeln, ihre eigene Zukunft gestalten zu können. Diese Überzeugung wird im Berufswahlkompetenzmodell als Verantwortung bzw. Kontrolle über das eigene Handeln bezeichnet. Die dritte motivationale Facette, die für eine positive berufliche Entwicklung bedeutsam ist, ist die Offenheit, die eigenen Interessen weiter $\mathrm{zu}$ erforschen und sich neuen beruflichen Entwicklungsperspektiven gegenüber nicht zu verschließen. Offenheit, im Sinne von Explorationsbereitschaft, befähigt Jugendliche folglich, die eigenen Interessen mit Tätigkeitsbeschreibungen verschiedener, gegebenenfalls neu kennengelernter Berufe abzugleichen. Die vierte, zu entwickelnde motivationale Facette ist die Zuversicht. Mit Zuversicht wird das Vertrauen der Jugendlichen in sich selbst beschrieben. Zuversichtliche Jugendliche sind bestärkt in ihrem Glauben, Hindernisse auf ihrem beruflichen Weg durch ihre eigenen Fähigkeiten erfolgreich bewältigen und ihre Zukunft durch ihr eigenes Handeln aktiv gestalten zu können (Driesel-Lange et al., 2020). Die motivationalen Einstellungen als Teil des Berufswahlkompetenzmodells basieren auf den vier Dimensionen der Adaptabilität (vgl. Abschnitt 5.2). Dies verdeutlicht einen weiteren Anknüpfungspunkt des Modells an internationale Theorien und Debatten.

\section{Die Handlungsdimension}

Neben dem berufswahlrelevanten Wissen und der Motivation, sich mit der eigenen Zukunft kognitiv und emotional zu beschäftigen, ist zudem die Handlungsfähigkeit entscheidend für einen gelungenen nachschulischen Übergang. Die erste Facette der Handlungsdimensionen, die Exploration, beschreibt die gezielte, systematische und handlungsbezogene Beschäftigung mit der eigenen beruflichen Zukunft. Denn im Gegensatz zu beruflich bereits Aktiven verfügen Jugendliche zu Beginn ihres Berufswahlprozesses über keine oder nur geringe Erfahrungen mit der Berufswelt, die sie für ihre beruflichen Entscheidungen nutzen können 
(Driesel-Lange et al., 2020). Umso bedeutender ist ihre gezielte Suche nach Informationen, die sie dann als Referenzrahmen und Grundlage ihrer beruflichen Entscheidungsprozesse heranziehen können. Die zweite Handlungsfacette, die Steuerung, bezeichnet die Fähigkeit, in den verschiedenen berufswahlrelevanten Kontexten, wie beispielsweise Bewerbungsgesprächen, situationsadäquat aufzutreten und zu reagieren. Die Selbststeuerung befähigt Jugendliche auch dazu, die gesetzten Ziele trotz möglicher Hindernisse oder Ablenkungen stringent zu verfolgen. Die Problemlösekompetenz als dritte Facette der Handlungsdimension befähigt Jugendliche, einen Plan bzw. Alternativszenarien zu entwickeln, um ein gefasstes berufliches Ziel erreichen zu können. Die vierte Facette der Handlungsdimension, das Stressmanagement, benötigen Jugendliche, um mit Schwierigkeiten wie Absagen im Bewerbungsprozess lösungsorientiert umgehen zu können (Driesel-Lange et al., 2020).

\subsection{Fragebogen zur Diagnostik von Berufswahlkompetenz}

Aus Gründen inhaltlicher Stringenz und Lesbarkeit wird dem empirischen Teil vorgegriffen und das Haupterhebungsinstrument der empirischen Studien in Kapitel 10 bis 12 bereits hier dargestellt. Der diagnostische Fragebogen - ThüBOMFragebogen zur Berufswahlkompetenz - wurde von Kaak et al. (2013) mit dem Ziel entwickelt, Berufswahlkompetenzstände und -entwicklungen Jugendlicher messen zu können. Die Messung von Kompetenzveränderungen ermöglicht gleichzeitig, berufsorientierende Maßnahmen hinsichtlich ihrer Effekte auf die Berufswahlkompetenzentwicklung Jugendlicher $\mathrm{zu}$ evaluieren und entsprechend adaptieren zu können (Kaak et al., 2013). In seiner ursprünglichen Form enthielt der Fragebogen zwölf Subskalen mit 143 Items, die die zwölf Berufswahlkompetenzfacetten des theoretischen Modells abbilden sollten (Kaak et al., 2013). Die Skalen wurden bereits etablierten Diagnostikinstrumenten wie dem EBwAFragebogen von Stangl und Seifert (1986) entlehnt und durch mehrere, neu entwickelte Items ergänzt. Auch die vier Dimensionen der Adaptabilität (siehe Abschnitt 5.2) wurden als Motivationsskalen integriert. Dabei basiert die Betroffenheitsskala u. a. auf Crites und Savickas (1995), während die drei anderen motivationalen Skalen Eigenentwicklungen von Kaak et al. (2013) darstellen. In einem iterativen Prozess wurde der Fragebogen auf 90 Items gekürzt und mit geringen sprachlichen Vereinfachungen, die dem besseren Verständnis dienen, veröffentlicht (Lipowski, Kaak, Kracke \& Holstein, 2015a). Aktuell befinden sich mehrere Kurzfragebögen in Testung. Die empirischen Studie in Kapitel 10, 11 
und 12 basieren jedoch auf dem etablierten Fragebogen mit 90 Items. Zur Messung des Berufswahlkompetenzstands bewerten die Teilnehmenden die Aussagen jeweils auf einer vierstufigen Likert-Skala. Die Tabelle 6.1 gibt einen Überblick über die zwölf Subskalen. Zudem verdeutlichen jeweils ein Beispielitem und die zugehörigen Antwortmöglichkeiten das Format des Instruments. Die Verwendung von Selbsteinschätzungen wird, wie die Autoren des Fragebogens selbst bemerken, in der Forschungsgemeinde aufgrund möglicher Verzerrungen mitunter kritisch bewertet, allerdings konnte Bühner (2009) eine positive Relation zwischen Selbstauskunft und dem eigentlichen Kompetenzstand belegen. Sedelmaier und

Tabelle 6.1 Fragebogen zur Diagnostik von Berufswahlkompetenz nach Kaak et al. (2013)

\begin{tabular}{|c|c|c|}
\hline Skala (N Items) & Beispielitem & \\
\hline Selbstwissen (9) & $\begin{array}{l}\text { Ich kann meine Fähigkeiten gut } \\
\text { einschätzen. }\end{array}$ & \multirow{4}{*}{$\begin{array}{l}1=\text { stimmt nicht } \\
2=\text { stimmt wenig } \\
3=\text { stimmt etwas } \\
4=\text { stimmt genau }\end{array}$} \\
\hline Konzeptwissen (6) & $\begin{array}{l}\text { Ich weiß, wie eine betriebliche } \\
\text { Berufsausbildung bzw. ein Studium } \\
\text { organisiert ist. }\end{array}$ & \\
\hline Bedingungswissen (7) & $\begin{array}{l}\text { Ich weiß genau, wie ich mich auf } \\
\text { meinen späteren Beruf vorbereiten } \\
\text { muss. }\end{array}$ & \\
\hline Planung \& Entscheidung (4) & $\begin{array}{l}\text { Ich habe mir für die nächste Zeit klare } \\
\text { Ziele gesetzt, um dem Berufseinstieg } \\
\text { näher zu kommen. }\end{array}$ & \\
\hline Betroffenheit (8) & $\begin{array}{l}\text { Es ist mir wichtig zu klären, für welche } \\
\text { Berufe ich mich eigne. }\end{array}$ & \multirow{3}{*}{$\begin{array}{l}1=\text { stimmt nicht } \\
2=\text { stimmt wenig } \\
3=\text { stimmt etwas } \\
4=\text { stimmt genau }\end{array}$} \\
\hline Eigenverantwortung (6) & $\begin{array}{l}\text { Ich mache mir jetzt schon viele } \\
\text { Gedanken darüber, was ich einmal } \\
\text { werden will. }\end{array}$ & \\
\hline Offenheit (7) & $\begin{array}{l}\text { Mir macht es Spaß, neue Berufe kennen } \\
\text { zu lernen. }\end{array}$ & \\
\hline Zuversicht (12) & $\begin{array}{l}\text { Wie sehr traust Du Dir zu, zu } \\
\text { beschreiben, wie Dein Wunschberuf } \\
\text { aussehen soll? }\end{array}$ & $\begin{array}{l}1=\text { gar nicht } \\
2=\text { wenig } \\
3=\text { eher } \\
4=\text { sehr }\end{array}$ \\
\hline Exploration (9) & $\begin{array}{l}\text { Wie oft hast Du in den letzten Monaten } \\
\text { Informationen über verschiedene } \\
\text { Berufe gesucht? }\end{array}$ & $\begin{array}{l}1=\text { gar nicht } \\
2=\text { wenig } \\
3=\text { eher } \\
4=\text { sehr }\end{array}$ \\
\hline
\end{tabular}


Tabelle 6.1 (Fortsetzung)

\begin{tabular}{l|l|l}
\hline Skala (N Items) & Beispielitem & \\
\hline Steuerung (7) & Wenn etwas, das ich tun wollte, & $1=$ stimmt nicht \\
& schiefgeht, will ich herausfinden, woran & $\begin{array}{l}\text { es gelegen hat. } \\
\text { estimmt wenig } \\
3=\text { stimmt etwas } \\
4=\text { stimmt genau }\end{array}$ \\
\cline { 1 - 2 } Problemlösen (10) & $\begin{array}{l}\text { Ich traue mir zu, eine Lösung zu finden, } \\
\text { wenn ich plötzlich in meinen }\end{array}$ & \\
& Schulleistungen absacken würde. & \\
\hline Stressmanagement (5) & An meinen späteren Beruf zu denken, & \\
& ist eine große Belastung für mich. & \\
\hline
\end{tabular}

Landes (2016) fanden zudem eine hohe Übereinstimmung zwischen der Selbstund Fremdeinschätzung von Kompetenzen, was wiederum auf die Validität von Selbstbewertungen hinweist.

\subsection{Auswahl des Instruments zur Erhebung von Berufswahlkompetenz}

Ein wesentliches Ziel dieser Arbeit ist es, die heterogene Bedarfslage Jugendlicher hinsichtlich der Unterstützung ihrer beruflichen Orientierung zu veranschaulichen und Profile ähnlicher Orientierungsbedarfe als mögliche Basis einer Binnendifferenzierung im schulischen Kontext zu identifizieren. In diesem Teilkapitel soll die Auswahl des Berufswahlkompetenzmodells von Driesel-Lange et al. (2010) als theoretische Basis zur Untersuchung der genannten Bedarfe transparent gemacht werden.

Um ein umfassendes Bild zur Heterogenität der berufswahlbedingten Bedarfe der Heranwachsenden zu generieren, bedarf es neben der Analyse der individuellen Voraussetzungen zweierlei. Zum einen ist eine Betrachtung des individuellen Stands bzw. Fortschritts im Berufswahlprozess erforderlich, um zu verstehen, welchen berufswahlrelevanten Aufgaben und Herausforderung die Heranwachsenden aktuell gegenüberstehen. Zum anderen bedarf es der Analyse des individuellen Entwicklungsstands berufswahlrelevanter Kompetenzen. Daraus lässt sich wiederum ableiten, inwieweit die zur Aufgabenbewältigung kritischen Fähigkeiten und Fertigkeiten bereits vorhanden oder noch zu erwerben sind. Folglich bedarf es für die empirischen Analysen in dieser Arbeit eines theoretischen Modells, das zur Beschreibung des komplexen, individuellen Prozesses verschiedene Perspektiven 
einnimmt, und dabei beispielsweise status- und geschlechtsbedingte Eingrenzungsprozesse (vgl. Boudon, 1974; Gottfredson, 1981, 2002) berücksichtigt. Die Annahme individueller Entwicklungsprozesse ist zudem als eine grundsätzliche Voraussetzung heterogener Entwicklungsverläufe bedeutsam. Des Weiteren sollte das theoretische Modell auf die berufliche Orientierung Jugendlicher anwendbar sein und möglichst den schulischen Kontext berücksichtigen. Wie gerade beschrieben, sollte das zugrundeliegende Modell Entwicklungsphasen, aus denen sich wiederum die zu lösenden Entwicklungsaufgaben ableiten lassen, berücksichtigen. Zuletzt stellt die Berücksichtigung von Entwicklungsvoraussetzungen, im Sinne benötigter Kompetenzen, einen weiteren Anspruch an das theoretische Modell dar.

Zur Herleitung der Auswahl des Berufswahlkompetenzmodells soll nach seiner detaillierten Vorstellung im vorangegangenen Teilkapitel (6.1) anhand der gerade beschriebenen Auswahlkriterien ein Vergleich zu den in Abschnitt 4.1 und 5.2 vorgestellten Modellen vorgenommen werden. In Abschnitt 4.1 wurden fünf Modelle erläutert, die den beruflichen Entwicklungsprozess, gänzlich oder mit Fokus auf einzelne Aspekte, in Phasen oder Sequenzen einteilen. Herzog et al. (2006) integrieren in ihrem Sechs-Phasenmodell, wie auch Driesel-Lange et al. (2010) in ihrem Berufswahlkompetenzmodell, multiple Perspektiven auf den beruflichen Orientierungsprozess, darunter Gottfredsons (1981, 2002) Eingrenzungsprozesse und den sozialen Lernprozess der Berufswahl (Butz, 2008b; Mitchell \& Krumboltz, 1990). Die anderen vier Entwicklungsmodelle fokussieren sich in der Beschreibung der jeweiligen Phasen vornehmlich auf eine Perspektive. Dies ist zu großen Teilen dem jeweiligen Fokus auf einzelne Bereiche des Berufswahlprozesses geschuldet. So betrachten beispielsweise Gati und Asher (2001a) in ihrem Drei-Stufen-Modell den Auswahl- und Konkretisierungsprozess beruflicher Optionen, sodass eine differenzialpsychologische Sicht, in der der Gedanke des Matchings zwischen Individuum und Beruf im Vordergrund steht, sinnvoll erscheint.

Alle vorgestellten Entwicklungsmodelle verbinden die identifizierten Phasen implizit oder explizit mit zu bewältigenden, phasentypischen Aufgaben. Mit Ausnahme von Pelka (2010a, 2010b) wird in Verbindung mit diesen Herausforderungen zudem der Erwerb bzw. das Vorhandensein von selbst- und berufswahlbezogenem Wissen und Kompetenzen impliziert (Fend, 1991; Gati \& Asher, 2001a; Herzog et al., 2006; Neuenschwander \& Hartmann, 2011). Jedoch werden die Kompetenzen ausschließlich im Berufswahlkompetenzmodell im Einzelnen benannt (vgl. Driesel-Lange et al., 2020). Auch in der Phaseneinteilung sowie im übergreifenden Verständnis des Berufswahlprozesses differieren die vorgestellten Modelle untereinander und im Vergleich zum Berufswahlkompetenzmodell. 
Während Driesel-Lange et al. (2010) entwicklungstypische Phasen postulieren, in denen verschiedene Facetten von Berufswahlkompetenz zur Anwendung kommen und gleichzeitig erworben werden (Driesel-Lange et al., 2020), handelt es sich bei Herzog et al. (2006) Sechs-Phasenmodell um eine Beschreibung von zwingend aufeinanderfolgenden Informations-, Eingrenzungs-, Entscheidungs- und Umsetzungsschritten. Neuenschwander und Hartmann (2011) nehmen in Hinblick auf den beruflichen Entwicklungsprozess wiederum einen diskontinuierlichen Verlauf an, in dem sie in ihrem Modell explizit optionale Wiederholungsphasen einzelner oder mehrerer Phasen integrieren und damit Unterschiede im beruflichen Entscheidungsprozess annehmen. Damit teilen Herzog et al. (2006) am ehesten die Auffassung der individuellen Entwicklungsverläufe von Driesel-Lange et al. (2010). Auch Gati und Asher (2001b) nennen die Möglichkeit einzelne Modellstufen zu wiederholen, beziehen sich dabei jedoch nur auf den Auswahl- und Entscheidungsprozess. Bis auf das gerade genannte Drei-Stufen-Pic-Modell (Gati \& Asher, 2001a, 2001b) beziehen sich alle vorgestellten Phasenmodelle auf die berufliche Orientierung im Jugendalter. Neuenschwander und Hartmann (2011) haben zudem explizit Bezug auf die berufliche Orientierung im Schweizer Schulsystem genommen, während das Berufswahlkompetenzmodell von Driesel-Lange et al. (2010) mit Blick auf die berufliche Orientierung im deutschen Schulsystem entwickelt wurde.

In Abschnitt 5.2 wurden drei Modelle vorgestellt, die ähnlich dem Berufswahlkompetenzmodell Kompetenzen und Ressourcen als Voraussetzungen beruflicher Entwicklung benennen. Hinsichtlich des Theoriebezugs und einzelner Unterkonstrukte zeigen sich mehrere Ähnlichkeiten zwischen den drei Modellen aus Abschnitt 5.2 und dem Berufswahlkompetenzmodell. Sowohl das Modell der Adaptabilität (Savickas, 1997, 2005), als auch die Modelle zu Karrierekompetenzen (Akkermans, Brenninkmeijer, et al., 2013) und Karriereressourcen (Hirschi, 2012; Hirschi et al., 2019) integrieren ähnlich dem Berufswahlkompetenzmodell zahlreiche Perspektiven. Gemeinsam ist den vier Modellen zudem die Annahme eines stetigen Gestaltungs- und damit Reaktionsbedarfs, der aus Veränderungen in der Arbeitswelt entsteht und sich wiederum in zu bewältigenden Aufgaben manifestiert. Jedoch bestehen in den Perspektiven, im Sinne von Zielsetzungen, auch Differenzen. Driesel-Lange et al. (2010) setzen sich mit dem Berufswahlkompetenzmodell zum Ziel, ein theoretisches Konstrukt als Ausgangsbasis theoriegeleiteter, evidenzbasierter Unterstützung der berufswahlbezogenen Entwicklung von Schüler*innen zu bieten (vgl. Driesel-Lange et al., 2020). Indes wurde das Karriereressourcen-Modell auch mit Blick auf die Praxis, jedoch zur Potentialentwicklung von Angestellten entwickelt (Akkermans, Brenninkmeijer et al., 2013). 
Wiederum verbindet die vier Modelle die Betonung von Eigenverantwortung und Eigeninitiative, die durch das Individuum aufgebracht werden müssen. Zugleich unterschieden sich die in Abschnitt 5.2 vorgestellten Modelle in ihrem Fokus auf die berufliche Entwicklung im Erwachsenenalter bzw. im Erwerbsleben vom Berufswahlkompetenzmodell. Bei Letzterem steht indes die Zeit vor dem Übergang in die Ausbildung bzw. das Studium im Vordergrund (DrieselLange et al., 2020). Das Konzept der Adaptabilität ist zwar grundsätzlich auf die Entwicklung im Jugendalter anwendbar, wie seine Integration in das Berufswahlkompetenzmodell unter Beweis stellt. Allerdings wurde das Instrument mit Blick auf die Beratung Erwerbstätiger oder Erwerbsfähiger entwickelt (Savickas, 2005). Dies spiegelt sich auch im Schwerpunkt der empirischen Forschung auf (junge) Erwachsene (Rudolph et al., 2017). Ebenso konzentrieren sich, in Kongruenz mit ihrem Modellziel, Akkermans, Brenninkmeijer, et al. (2013) auf Angestellte. Hirschi und Kolleg*innen befinden sich zwar in der Validierungsphase eines Diagnostikinstruments für Schüler*innen, jedoch liegt der Fokus des theoretischen Modells auf der Erwerbszeit (Hirschi, 2012; Hirschi et al., 2019). Viele Überschneidungen bestehen zwischen den vier Modellen hinsichtlich der Relevanz von Wissen, Fähigkeiten und Fertigkeiten. Ein besonders bedeutsamer Bezug ergibt sich zwischen dem Konstrukt der Adaptabilität und dem Berufswahlkompetenzmodell, in dem die vier von Savickas (2005) benannten Facetten der Adaptabilität als motivationale Facetten integriert sind (Driesel-Lange et al., 2020). Gleichzeitig wird in keinem der drei vorgestellten Modelle die Entwicklung der genannten Fähigkeiten, motivationalen Einstellungen oder Ressourcen in den Kontext spezifischer Phasen gestellt.

Die vorangegangene Diskussion zusammenfassend, besteht die größte Nähe zwischen dem Berufswahlkompetenzmodell von Driesel-Lange et al. (2010) und dem entwicklungsbezogenen Sechs-Phasenmodell von Herzog et al. (2006) einerseits und Hirschis (2012) Karriereressourcen-Modell andererseits. Das SechsPhasenmodell von Herzog et al. (2006) nähert sich in seiner langfristigen Betrachtung des Berufswahlprozesses, dem Fokus auf das Jugendalter und der Integration verschiedener Perspektiven und Theorien dem Berufswahlkompetenzmodell. Jedoch unterscheiden sich die beiden Modelle in zwei wesentlichen Punkten, das Berufswahlkompetenzmodell nimmt expliziten Bezug auf den schulischen Kontext und formuliert konkrete Berufswahlkompetenzfacetten, die wiederum mit den Phasen und ihren Aufgaben verzahnt werden. Das Karriereressourcen-Modell von Hirschi (2012) wiederum formuliert konkrete Ressourcen und Kompetenzen, die eine erfolgreiche berufliche Entwicklung bedingen, allerdings wird der Prozess, in dem die Heranwachsenden diese Fähigkeiten und Ressourcen über 
entsprechende Aufgaben erwerben, weder konkretisiert, noch in fassbare Phasen eingeteilt. Besonders in der Verzahnung der beiden Perspektiven - sowohl phasen- als auch kompetenzbezogene Bedingungen im Kontext beruflicher Entwicklung zu adressieren - sowie in der Fokussierung auf Jugendliche innerhalb des deutschen sekundären Bildungssektors liegen die Stärken des vorgestellten Modells. Damit bildet dieses Modell ein starkes theoretisches Fundament für weitere empirische Untersuchungen, die mit dem Vorhandensein des diagnostischen Instruments von Kaak et al. (2013) (vgl. auch Teilkapitel 6.2) eine direkte empirische Überprüfung der Berufswahlkompetenzentwicklung und bestehender Unterschiede ermöglicht.

Open Access Dieses Kapitel wird unter der Creative Commons Namensnennung 4.0 International Lizenz (http://creativecommons.org/licenses/by/4.0/deed.de) veröffentlicht, welche die Nutzung, Vervielfältigung, Bearbeitung, Verbreitung und Wiedergabe in jeglichem Medium und Format erlaubt, sofern Sie den/die ursprünglichen Autor(en) und die Quelle ordnungsgemäß nennen, einen Link zur Creative Commons Lizenz beifügen und angeben, ob Änderungen vorgenommen wurden.

Die in diesem Kapitel enthaltenen Bilder und sonstiges Drittmaterial unterliegen ebenfalls der genannten Creative Commons Lizenz, sofern sich aus der Abbildungslegende nichts anderes ergibt. Sofern das betreffende Material nicht unter der genannten Creative Commons Lizenz steht und die betreffende Handlung nicht nach gesetzlichen Vorschriften erlaubt ist, ist für die oben aufgeführten Weiterverwendungen des Materials die Einwilligung des jeweiligen Rechteinhabers einzuholen. 